\title{
Analysis of flexible structures with occasionally rigid parts under transient loading
}

\author{
B. Göttlicher, K. Schweizerhof * \\ Institut für Mechanik, University Karlsruhe, 76128 Karlsruhe, Germany \\ Received 5 August 2003; accepted 21 March 2005 \\ Available online 31 May 2005
}

\begin{abstract}
In the computation of solid structures under long duration transient loading it is often advisable to treat parts of the structure at least for some time of the analysis as rigid bodies. Such a procedure increases first the efficiency of the analysis considerably and second the numerical condition of the system of equations benefits substantially within the numerical solution process. In particular structural modeling capabilities concerning boundary conditions and load transfer are considerably extended compared to a straightforward modeling with rigid bodies. The parts assumed rigid with small strains and sharing almost only a rigid body motion can be found in systems with high difference in stiffness as well as in systems with modestly loaded parts.

A methodology based on the Energy Momentum Method is developed for creating occasionally rigid bodies within the computation. A criterion based on strain rates is used to decide for the modification from flexible to rigid. For optimal reduction of the number of degrees of freedom the rigid bodies are formulated using a transformation to minimal coordinates, also called master slave concept. The numerical examples involving long duration motion and large rotations demonstrate the possibilities of the developed procedure.
\end{abstract}

(C) 2005 Elsevier Ltd. All rights reserved.

Keywords: Rigid body mechanics; Coupling with finite elements; Structural dynamics; Modification rigid to flexible to rigid

\section{Introduction}

In the computation of solid structures under long duration transient loading it is often advisable to treat parts of the flexible structure at least for some time of the analysis as rigid. Such a procedure increases first the overall efficiency of the analysis considerably and

\footnotetext{
* Corresponding author.

E-mail address: karl.schweizerhof@bau-verm.uni-karlsruhe. de (K. Schweizerhof).

URL: http://www.ifm.uni-karlsruhe.de (K. Schweizerhof).
}

second the numerical condition of the system of equations benefits substantially within the numerical solution process. The modeling as a rigid body has also more advantages than an increase of the mesh coarseness, as with a rigid body the boundary conditions and the distribution of the masses can be further modeled with the accuracy of the fine mesh whereas the final number of degrees of freedom is considerably reduced. The parts assumed to become rigid with small strains and sharing almost only a rigid body motion can be found in systems with large differences in stiffness as well as in systems with modestly loaded parts.

Furthermore, the identification and visualization of parts that behave almost as rigid bodies improve the 
understanding of the basic dynamic behavior of a structure. Suitable continuous flexible structures can be reduced to simple multibody structures with the help of interim results. In such a case the transition from the originally completely deformable structure model to the simplified rigid body model depends also on the external load. The automatic setting to rigid can be used particularly for systems in which the boundary conditions (neither by external loading nor by support) do not change too frequently.

The general procedure allows a complete adaptation of the simulation model that can partially also be used for an optimization of the structure. The method itself represents in this case a combination of the Finite Element Method and Rigid Body Dynamics. Thus the points of interest concerning the proper selection resp. definition as 'rigid' parts are:

First: The detection of parts that are qualified to be simplified to rigid bodies within the analysis of the transient problem.

Second: The reduction of the data describing the behavior of the actual flexible part to the data describing the rigid body behavior.

Within the analysis, the rigid bodies are modeled by reducing all data describing the motion of the corresponding flexible structural part to the degrees of freedom of a rigid body. Therefore the structural parts to be set to rigid are 'frozen' at the actual configuration. The fulfillment of linear and angular momentum should be guaranteed when switching to rigid. However, there is no perfect energy conservation possible in the process as the model changes.

The necessary coupling can be realized by Penalty-, Augmented Lagrange- or Lagrange Multiplier methods which all have in common that the number of degrees of freedom is mostly not optimally reduced and in addition problems concerning the condition of the equation systems are introduced. Most of the cited problems can be avoided by direct transformation to minimal coordinates using nonlinear constraint conditions as described in [16]. Of vital importance is also a proper treatment of the combined problem within the numerical time integration.

The coupling of rigid and flexible structures in the socalled Energy Momentum Method using the Penalty procedure is described in [3-5]. A corresponding procedure using Lagrange multipliers was worked out in detail in $[2,15]$ and can also be found in [10]. The latter article includes an efficient formulation for the integration of general constraint conditions on the basis of minimal coordinates that are explained more closely in [8]. For an extensive discussion we also refer to Göttlicher [6].

Because of the additional transformation work required for the reduction i.e. for reasons of numerical effi- ciency, it is not recommended to set parts to rigid that consist of individual or only few elements. Further the setting to rigid of suitable parts of the structure might produce configurations where rigid bodies are linked to each other kinematically i.e. with joints of different formation. As modeling the pertaining kinematics requires quite laborious calculations, the formation of kinematic rigid body chains should be avoided by omitting to set parts of the structure to rigid that are within the part of kinematic couplings. In this case, the rigid bodies have no direct connection.

As time integration scheme the 'Energy Momentum Method' proposed by Simo et al. (see e.g. [13,14]) or the implicit midpoint rule are suited, as it is important for an efficient procedure, that accelerations are not part of the weak form. Within the current contribution, we will focus on the 'Energy Momentum Method'. Its property of fulfilling the conservation laws within the time step makes it particularly suitable for long duration analysis. If damping is introduced, which is advisable to avoid high frequency effects, the numerical damping scheme proposed by Armero and Petöcz [1] provides linear and angular momentum conservation. An expansion to finite rotations of beams is presented in [9].

The flexible structures are modeled with displacement based 3D continuum elements. Besides 3D-volume elements, the so called 'solid-shell' elements (see e.g. [7]), based on an enhancement of the strain, can be used for the structural discretization, as they are formulated with the same degrees of freedom. The formulation for so-called enhanced elements within the Energy Momentum Method is generally described in [12]). The motion of the generated 3-D rigid bodies is described as usual with three translational and three rotational degrees of freedom. The modeling with arbitrarily connected flexible and rigid parts allows the handling of geometrically nonlinear problems with arbitrarily large rotations. Finally, the application of the proposed modeling and integration scheme is discussed using representative numerical examples on the basis of geometrically nonlinear $3 \mathrm{D}$ continuum problems.

The outline of the paper is as follows: In Section 2, the time integration of the coupled structure with the Energy Momentum Method is presented using a transformation on minimal coordinates. In Section 3, the procedure for the automatic setting to rigid of a suitable flexible part of the structure is outlined. In Section 4, instructions for the calculation of the mass, for the center of mass and for the inertia tensor of the parts of the structure to be set to rigid are given. In Section 5, the computation of the mass center velocities of the parts of the structure that are to be set to rigid while conserving the linear and angular momentum is discussed. In Section 6, a method is proposed that permits the realization of energy conservation for the complete process 
throughout a repetitive process of setting to rigid and resetting to flexible. In Section 7, numerical examples with special emphasis on the fulfillment of the conservation laws and an overview over a complete modification process from flexible to rigid and back to flexible are given. Section 8 contains some concluding remarks.

\section{Time integration of the coupled structure}

As time integration scheme the so-called 'Energy Momentum Method' proposed by Simo et al. (see e.g. $[13,14])$ is chosen. In the following section we describe the time integration of the coupled rigid flexible system in minimal coordinates with the Energy Momentum Method with a specific reference to Zienkiewicz and Taylor [16], Ibrahimbegovic et al. [10] and Ibrahimbegovic and Mamouri [8]. The formulation of the weak form requires the expression of the displacement degrees of freedom $\boldsymbol{u}^{g}$ of the nodes of those flexible elements that are directly adjacent to rigid bodies (in global coordinates) by the translational and rotational degrees of freedom of the rigid body $\boldsymbol{r}^{s}$ and $\boldsymbol{\theta}^{s}$ for the discretized structure in accordance with Fig. 1. Thus, the following constraint conditions concerning displacements are required for e.g. node $i$ :

$\boldsymbol{z}_{i}:=\boldsymbol{r}_{i}+\left(\boldsymbol{R} \boldsymbol{V}_{i}-\boldsymbol{V}_{i}\right)-\boldsymbol{u}_{i}^{g}=\mathbf{0}$.

With $\boldsymbol{R}$ as the rotation tensor of the corresponding rigid body and $\boldsymbol{V}_{i}$ the connection vector at the beginning of the analysis at time 0 .

In addition, the time derivative of Eq. (1) is required for proper transformation of the mass matrix. This is the constraint condition with regard to the velocities:

$\dot{\boldsymbol{z}}_{i}:=\dot{\boldsymbol{r}}_{i}+\dot{\boldsymbol{R}} \boldsymbol{V}_{i}-\dot{\boldsymbol{u}}_{i}^{g}=\mathbf{0}$,

$\dot{\boldsymbol{r}}_{i}+\boldsymbol{\omega} \times \boldsymbol{R} \boldsymbol{V}_{i}-\dot{\boldsymbol{u}}_{i}^{g}=\mathbf{0}$

with the vector of the angular velocities $\omega$.
For the description of the coupling within one-step time integration procedures, the connection vector is assumed from the center of mass of the rigid body to the coupling node $i$ in the time step as arithmetical mean of its components at the beginning of the time step (index $n$ ) and at the end of the time step (index $n+1$ ).

$\boldsymbol{v}_{i, \mathrm{av}}=\frac{1}{2}\left(\boldsymbol{v}_{i, n}+\boldsymbol{v}_{i, n+1}\right)=\boldsymbol{R}_{\mathrm{av}} \boldsymbol{V}_{i}$.

Accordingly, the rotation tensor describing the spatial rotation of the rigid body is defined as follows:

$\boldsymbol{R}_{\mathrm{av}}=\frac{1}{2}\left(\boldsymbol{R}_{n}+\boldsymbol{R}_{n+1}\right)$.

These pre-calculations lead to the virtual form of the constraint conditions for the coupling of the node $i$ to the rigid body $j$ as follows

$\delta \boldsymbol{u}_{i}=\delta \boldsymbol{r}_{j}-\boldsymbol{v}_{i, \mathrm{av}} \times \delta \boldsymbol{\theta}_{j}=\delta \boldsymbol{r}_{j}-\hat{\boldsymbol{v}}_{i, \mathrm{av}} \delta \boldsymbol{\theta}_{j}$

with $\hat{\boldsymbol{v}}_{i, \text { av }}$ defined as the skew symmetric tensor to $v_{i, \text { av }}$ as

$\hat{\boldsymbol{v}}_{i, \mathrm{av}}=\left[\begin{array}{ccc}0 & -v_{i, \mathrm{av}, 3} & v_{i, \mathrm{av}, 2} \\ v_{i, \mathrm{av}, 3} & 0 & -v_{i, \mathrm{av}, 1} \\ -v_{i, \mathrm{av}, 2} & v_{i, \mathrm{av}, 1} & 0\end{array}\right]$.

Only this approach allows the fulfillment of linear and angular momentum and energy conservation within a time step, see Chen [2] for the pertaining proofs.

In order to be able to formulate the virtual operations, as shown in Fig. 1, the vector of the degrees of displacement and/or rotation freedom $\boldsymbol{u}$ is split into

$\boldsymbol{u}^{u}=\left[\boldsymbol{u}_{1}^{u}, \ldots, \boldsymbol{u}_{n_{u}}^{u}\right]$,

$\boldsymbol{u}^{g}=\left[\boldsymbol{u}_{1}^{g}, \ldots, \boldsymbol{u}_{n_{g}}^{g}\right]$,

$\boldsymbol{r}^{s}=\left[\boldsymbol{r}_{1}^{s}, \boldsymbol{\theta}_{1}^{s}, \ldots, \boldsymbol{r}_{n_{s}}^{s}, \boldsymbol{\theta}_{n_{s}}^{s}\right]$.

In this connection $\boldsymbol{u}^{u}$ is composed of the $n_{u}$ node displacement vectors of the uncoupled flexible parts of the structure (not adjacent to a rigid body), $\boldsymbol{u}^{g}$ is composed of the $n_{g}$ nodal displacement vectors of the flexible parts

\section{Degrees of freedom of}
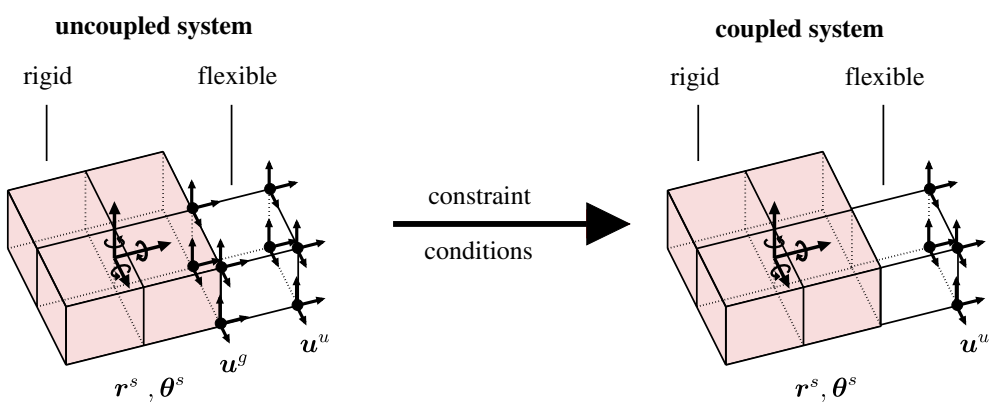

Fig. 1. 3D continuum with partially rigid part. Reduction of degrees of freedom by coupling with minimal coordinates. $\boldsymbol{u}^{u}$ displacement degrees of freedom for nodes of flexible elements. 
of the structure, directly located at the boundary of the rigid body parts, and $\boldsymbol{r}^{s}$ is composed of the $n_{s}$ displacement and rotation vectors of the rigid bodies.

The momentum vector

$\boldsymbol{p}=\left[\boldsymbol{p}_{1}, \ldots, \boldsymbol{p}_{n_{f}}\right]=\boldsymbol{M} \dot{\boldsymbol{u}}$

can analogously be split into the vectors $\boldsymbol{p}^{u}, \boldsymbol{p}^{g}$ and $\boldsymbol{p}^{s}$.

The weak form of the momentum balance for the unloaded, coupled system discretized in space and time leads for the uncoupled $(u)$, coupled $(g)$ and rigid parts $(s)$ to the following results:

$\delta \Pi^{u}+\delta \Pi^{g}+\delta \Pi^{s}=0$

with the corresponding parts of the virtual form

$$
\begin{aligned}
\delta \Pi^{u} \Delta t= & \left(\boldsymbol{p}_{n+1}^{u}-\boldsymbol{p}_{n}^{u}\right) \cdot \delta \boldsymbol{u}^{u}+\Delta t \boldsymbol{f}_{n+\frac{1}{2}}^{u} \cdot \delta \boldsymbol{u}^{u}, \\
\delta \Pi^{g} \Delta t= & \left(\boldsymbol{p}_{n+1}^{g}-\boldsymbol{p}_{n}^{g}\right) \cdot \delta \boldsymbol{u}^{g}+\Delta t \boldsymbol{f}_{n+\frac{1}{2}}^{g} \cdot \delta \boldsymbol{u}^{g}, \\
\delta \Pi^{s} \Delta t= & \sum_{j=1}^{n_{s}}\left\{\boldsymbol{M}_{j}^{s}\left(\dot{r}_{j, n+1}^{s}-\dot{r}_{j, n}^{s}\right) \cdot \delta \boldsymbol{r}_{j}^{s}\right. \\
& \left.+\left(\boldsymbol{I}_{j, n+1}^{s}, \boldsymbol{w}_{j, n+1}^{s}-\boldsymbol{I}_{j, n}^{s} \boldsymbol{w}_{j, n}^{s}\right) \cdot \delta \boldsymbol{\theta}_{j}^{s}\right\} .
\end{aligned}
$$

In the portions of the virtual operations for each node $i$ coupled to the rigid body $j$, the displacements of the nodes $i$ can be eliminated using the constraint condition (5) as follows:

$\boldsymbol{f}_{i, n+\frac{1}{2}}^{g} \cdot \delta \boldsymbol{u}_{i}^{g}=\boldsymbol{f}_{i, n+\frac{1}{2}}^{g} \cdot \delta \boldsymbol{r}_{j}^{s}+\left(\boldsymbol{v}_{i, \mathrm{av}} \times \boldsymbol{f}_{i, n+\frac{1}{2}}^{g}\right) \cdot \delta \boldsymbol{\theta}_{j}^{s}$,

$\boldsymbol{p}_{i}^{g} \cdot \delta \boldsymbol{u}_{i}^{g}=\boldsymbol{p}_{i}^{g} \cdot \delta \boldsymbol{r}_{j}^{s}+\left(\boldsymbol{v}_{i, \mathrm{av}} \times \boldsymbol{p}_{i}^{g}\right) \cdot \delta \boldsymbol{\theta}_{j}^{s}$.

In the above equations, $\dot{\boldsymbol{r}}_{s}$ and $\boldsymbol{w}_{s}$ refer to the translational and rotational velocity vectors of the rigid bodies centers of mass, $\boldsymbol{M}_{s}=m^{s}, \mathbf{1}_{3 \times 3}$ refers to the (diagonal) mass matrix and $\boldsymbol{I}_{s}$ to the mass inertia tensor of the rigid body. $\boldsymbol{f}^{u}$ and $\boldsymbol{f}^{g}$ refer to the nodal vectors of the residual forces of the uncoupled resp. coupled degrees of freedom, evaluated in the middle between time $n$ and $n+1$.

\subsection{Simplification for diagonal mass matrices}

If the mass matrices of the flexible elements that are adjacent to rigid bodies have diagonal structures, the mass matrices of the rigid parts of the structure (including the nodes coupled to them) and of the flexible parts are consequently uncoupled in the mass matrix also after the transformation to minimal coordinates. In the following we will take a look at the influence of the transformation on the mass matrix at the end of the time step. The nodal mass $m_{i}$ is coupled to the mass matrix of the (uncoupled) rigid body $j$ by means of the constraint condition (14). The mass matrix of the rigid body is defined as:

$$
\boldsymbol{M}_{n+1}^{s, u}=\left(\begin{array}{cc}
m_{j}^{s} \mathbf{1}_{3 \times 3} & \mathbf{0}_{3 \times 3} \\
\mathbf{0}_{3 \times 3} & \mathbf{I}_{j, n+1}^{s}
\end{array}\right)
$$

with the identity matrix $\mathbf{1}_{3 \times 3}$. The introduction of the momentum vector

$$
\begin{aligned}
\boldsymbol{p}_{i, n+1}^{g} & =m_{i} \dot{\boldsymbol{u}}_{i, n+1}^{g} \\
& =m_{i}\left(\dot{\boldsymbol{r}}_{j, n+1}^{s}+\hat{\boldsymbol{v}}_{i, n+1}^{\mathrm{T}} \boldsymbol{\omega}_{j, n+1}^{s}\right) \quad \text { because of (2) }
\end{aligned}
$$

in (14) leads to

$$
\begin{aligned}
\boldsymbol{p}_{i, n+1}^{g} \cdot \delta \boldsymbol{u}_{i}^{g}= & m_{i} \dot{\boldsymbol{r}}_{j, n+1}^{s} \cdot \delta \boldsymbol{r}_{j}^{s}+m_{i} \hat{\boldsymbol{v}}_{i, n+1}^{\mathrm{T}} \boldsymbol{\omega}_{j, n+1}^{s} \cdot \delta \boldsymbol{r}_{j, n+1}^{s} \\
& +m_{i} \hat{\boldsymbol{v}}_{i, \mathrm{av}} \dot{\boldsymbol{r}}_{j, n+1}^{s} \cdot \delta \boldsymbol{\theta}_{j}^{s}+m_{i} \hat{\boldsymbol{v}}_{i, \mathrm{av}} \hat{\boldsymbol{v}}_{i, n+1}^{\mathrm{T}} \boldsymbol{\omega}_{j, n+1}^{s} \cdot \delta \boldsymbol{\theta}_{j}^{s} .
\end{aligned}
$$

If this is inserted in (9), the enhanced mass matrix of the rigid body is found as

$$
\boldsymbol{M}_{n+1}^{s, g}=\left(\begin{array}{cc}
\left(m_{j}^{s}+m_{i}\right) \mathbf{1}_{3 \times 3} & m_{i} \hat{\boldsymbol{v}}_{i, n+1}^{\mathrm{T}} \\
m_{i} \hat{\boldsymbol{v}}_{i, \mathrm{av}} & m_{i} \hat{\boldsymbol{v}}_{i, \mathrm{av}} \hat{\boldsymbol{v}}_{i, n+1}^{\mathrm{T}}+\boldsymbol{I}_{j, n+1}^{s}
\end{array}\right) .
$$

The coupling terms between the mass portions for displacements and rotations result from the fact that the position of the rigid bodies center of mass changes as a consequence of the coupling of a mass to the rigid body. When using the constraint condition (14), the center of mass of the original rigid body is continued to be used as reference point, as it is done here.

\subsection{Numerical damping}

In the Energy Momentum Method [13], the weak form of the momentum balance in the time step $t_{n} \rightarrow t_{n+1}$ for an unloaded and undamped structure becomes:

$\frac{1}{\Delta t} \int_{B_{0}} \varrho_{0}\left(\dot{\boldsymbol{u}}_{n+1}-\dot{\boldsymbol{u}}_{n}\right) \cdot \delta \boldsymbol{u} \mathrm{d} V+\int_{B_{0}} \boldsymbol{F}_{\frac{1}{2}} \boldsymbol{S}_{\mathrm{av}}: \operatorname{grad} \delta \boldsymbol{u} \mathrm{d} V=\mathbf{0}$

with

$\boldsymbol{F}_{\frac{1}{2}}=\boldsymbol{F}\left(\boldsymbol{u}_{\frac{1}{2}}\right)$,

$\boldsymbol{u}_{\frac{1}{2}}=\frac{1}{2}\left(\boldsymbol{u}_{n}+\boldsymbol{u}_{n+1}\right)$,

$\boldsymbol{S}_{\mathrm{av}}=\frac{1}{2}\left(\boldsymbol{S}_{n+1}+\boldsymbol{S}_{n}\right)$.

In these equations, $\boldsymbol{F}$ is the deformation gradient, $\boldsymbol{S}$ the second Piola Kirchhoff stress tensor, $\varrho_{0}$ the density and $\boldsymbol{u}$ the displacement vector. This approach allows to prove linear and angular momentum as well as energy conservation in the time step (see [13]) for St. VenantKirchhoff material law.

As an extension, Armero and Petöcz [1] proposed the following form to control energy dissipation by a modification of the second PK stress tensor introduced then in (21), fulfilling linear and angular momentum conservation:

$\boldsymbol{S}=\boldsymbol{S}_{\mathrm{da}}=\left[\left(\frac{1}{2}-\xi\right) \boldsymbol{S}_{n}+\left(\frac{1}{2}+\xi\right) \boldsymbol{S}_{n+1}\right]$.

For positive values of $\xi$ energy is dissipated in particular for the higher modes. 


\section{Procedure for the automatic setting of flexible parts to rigid}

The identification of flexible parts that can be set to rigid requires the observation of the behavior of the structure throughout a certain period of time. For an efficient algorithm the time interval $I=\left[t_{0}, t_{0}+T_{\text {ges }}\right]$ to be integrated is subdivided into a sequence of $N_{\text {red }}$ time intervals $T_{\text {red }_{n}}=t_{n}-t_{n-1}$ that consist for their part of $M_{\text {red }}$ time intervals $\Delta t_{m}=t_{m}-t_{m-1}$ (of the time discretization for the solution of the motion equation) resulting in

$$
\begin{aligned}
\int_{t_{0}}^{t_{0}+T_{\text {ges }}}[\ldots] \mathrm{d} t & =\sum_{n=1}^{N_{\text {red }}} \int_{t_{n-1}}^{t_{n}}[\ldots] \mathrm{d} t \\
& =\sum_{n=1}^{N_{\text {red }}} \sum_{m=1}^{M_{\text {red }}} \int_{t_{m-1}}^{t_{m}}[\ldots] \mathrm{d} t .
\end{aligned}
$$

Within each time interval $T_{\text {red }}$, the behavior of the structure is checked in each time step $\Delta t$ on compliance with a criterion that will be introduced in the following sections. At the end of the time interval, those flexible parts of the structure are set to rigid that have always complied with the criterion throughout the time interval.

If the boundary conditions (Dirichlet or Neumann) should change, after individual parts have been set to rigid, the rigid body system obtained up to this point in time is in general not suitable for further analyses. As the influence of such a change on the system can hardly be estimated a priori, all rigid bodies have to be dissolved in this case and the computation has to be repeated for the time step in which the changes have occurred. Afterwards, the algorithm of the setting to rigid can be resumed. The course of the procedure is presented in the flow diagram Fig. 2, and will be discussed in detail in the following sections.

\subsection{Pre-conditions concerning the observation period $T_{\text {red }}$}

The choice of $T_{\text {red }}$ should provide for the possibility that the most undesirable event (greatest deviation from the behavior of a rigid body) for the selected criterion might occur during the observation period. This ensures adequate conditions as with periodical vibration processes for example, $T_{\text {red }}$ must be greater than the duration of the period. As the examined processes are in general not periodical and, as the duration of the period is often unknown, even in case of periodical processes, we can only recommend the fulfillment of the following necessary conditions:

The value selected for the observation period $T_{\text {red }}$ should be larger than the duration of the period pertaining

(a) to the lowest (exited) natural frequency or

(b) to the lowest frequency of a modification of any boundary condition.

\subsection{Criterion for the identification of flexible parts that behave like rigid bodies}

A possible rigid body criterion to check the relative deformations of a deformable body is: A part of the structure is considered as a rigid body if the changes in its geometry during the observed time interval $T_{\text {red }}$ are sufficiently small. The changes in geometry can be controlled via the changes of the strains.

Accordingly, the normalized strain rate

$\mathbf{e}^{e}=\frac{\sqrt{\int\left(\mathbf{E}_{n+1}-\mathbf{E}_{n}\right):\left(\mathbf{E}_{n+1}-\mathbf{E}_{n}\right) \mathrm{d} V_{0}^{e}}}{\Delta t V_{0}^{e}} \leqslant$ tol

is computed in each time interval for each flexible Finite Element. In this equation, $\mathbf{E}_{n}, \mathbf{E}_{n+1}$ are the Green strain tensors at the beginning and at the end of the time step, $V_{0}^{e}$ refers to the initial element volume at time 0 . The criterion is objective, i.e. independent of the rotations of the rigid body. With respect to the discretization in space and time, a dependency exists only so far as the mean of the strain changes has to be taken within the selected part of the structure and the selected time domain. The criterion can be controlled very efficiently as the values to build the element stiffness matrices and residual vectors have to be computed anyway. Merely the computation of the integral, of which the weighting factors have to be determined anyway, is required in addition. All elements that do not comply with this criterion in any time step within $T_{\text {red }}$ are marked. After the end of the time interval $T_{\text {red }}$ rigid bodies can be build up from all elements that are not marked.

\subsection{Modification due to loading or contact of rigid bodies}

It should however be retained that the described procedure is not suitable to verify whether the reduction of kinematics to the values of a rigid body after the setting to rigid of a flexible part of the structure is admissible within a continuing analysis. Only the entirely flexible model is generally valid for the comprehensive determination of general changes of the loading or contact/ impact with other bodies, though even then the discretization should be adjusted. As internal stresses and deformations cannot be computed for rigid bodies, the model modified with rigid body parts looses its general validity. Therefore, the parts previously set to rigid have to be transformed back into flexible parts as is also shown in the flow diagram. However, then we have to note that even, when the full relative velocity distribution for the obtained rigid bodies is stored in the analysis, there is no unique way to regain the correct phase of the vibration. 


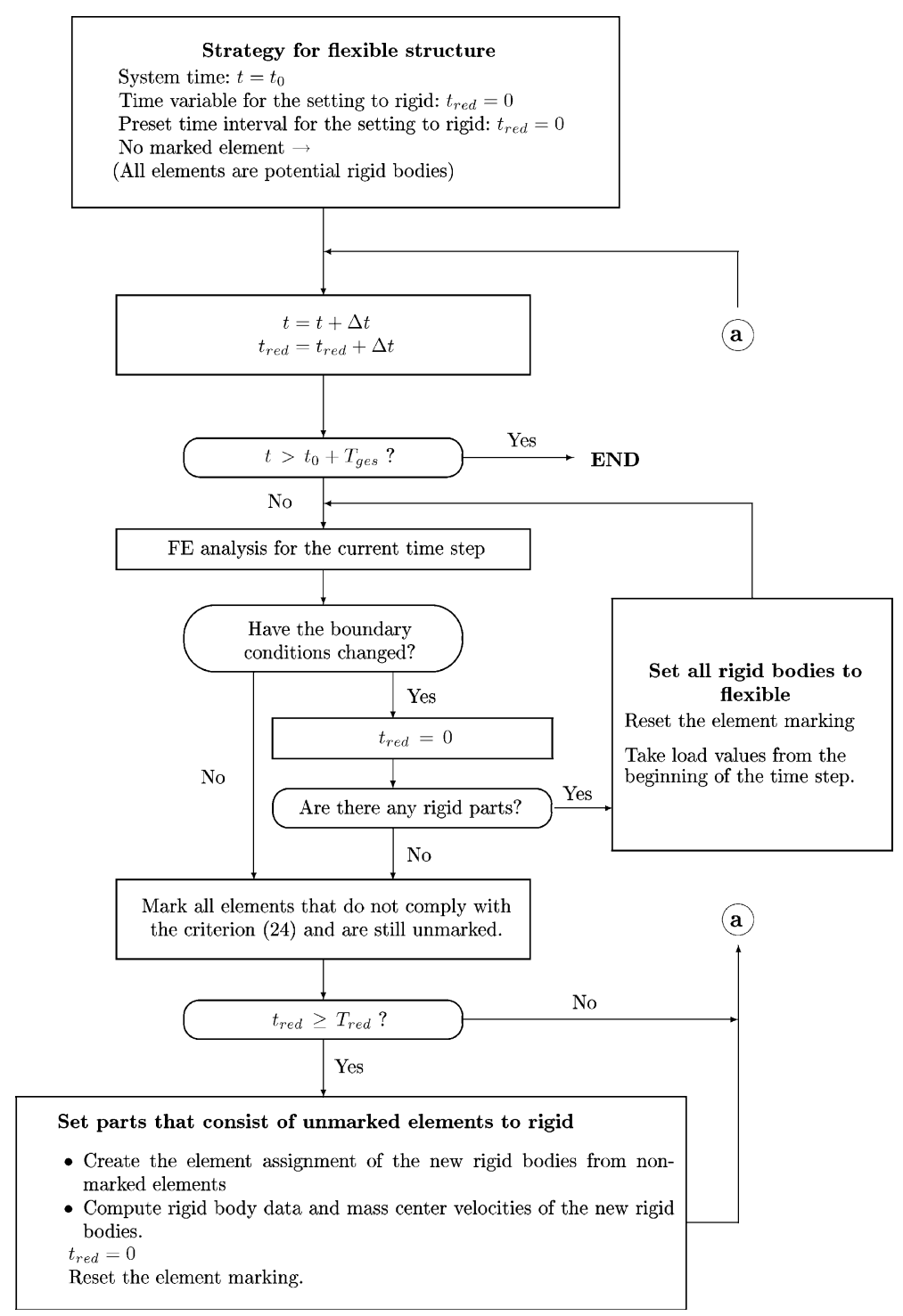

Fig. 2. Flow chart of a controlled setting of flexible parts to rigid.

\section{Rigid body data of the structure parts to be set to rigid}

As the parts to be set to rigid might contain strains that could be significant for other subsequent analyses e.g. they lead to a large deformation changing the shape of the body considerably and maybe important for a modified loading later, they are frozen in the current configuration. By doing so the number of degrees of freedom of each rigid part is reduced to 6 degrees of freedom of the rigid body as shown in Fig. 3. The information on the state of strain has to be stored immediately before the setting to rigid. In addition, further required variables e.g. for the description of nonlinear material behavior should also be stored. In case the parts should be reset to flexible, their portion of the strain energy of the total structure can be reconstructed in a unique fashion different from the velocity terms. This ensures that the analysis with the original FE mesh can be continued without problems concerning the strain energy.

We assume in the following sections that the flexible parts are computed either completely with a consistent mass matrix or completely with a diagonalized mass matrix. It is not meaningful in this case to achieve the decoupling of the rigid bodies mass matrices by assuming a diagonalized mass matrix for the adjacent flexible elements only, whereas for the remaining structure a 

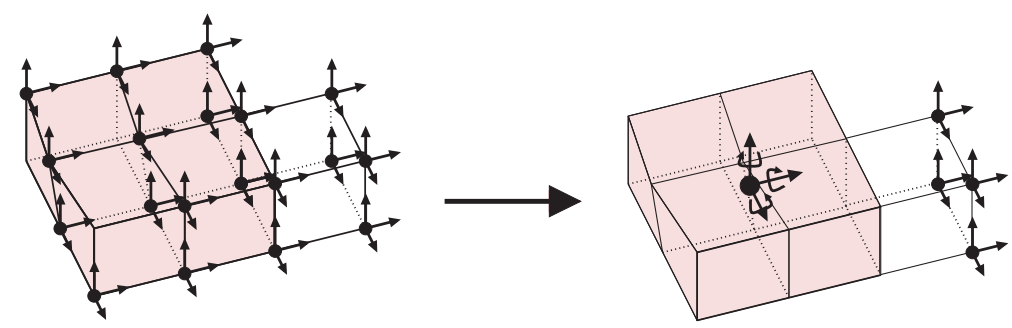

Fig. 3. Reduction of degrees of freedom after setting of parts to be rigid.

consistent formulation will be used, because it is not known in advance which parts will become rigid in the course of the examination. In addition, the model is not allowed to be modified in this respect during the complete analysis, as this would otherwise affect the angular momentum conservation.

In order to satisfy the angular momentum conservation, the computation of the rigid body data of previously flexible parts should always be based on the same distribution of masses (consistent or lumped) as the computation of the mass matrices of the flexible elements.

If in the course of an analysis the automatic setting to rigid is carried out, it should be assumed that some rigid parts may have existed before. Thus the rigid bodies for the subsequent analysis will therefore in general not be identical to the existing ones. Any change will only lead to an increase of the rigid parts as the proposed procedure will never lead to the dissolution of rigid parts except when the boundary conditions are changed. It may also happen that previously separated rigid bodies are combined. This means that in case of any modification the rigid body data of the rigid parts have to be recomputed from the original FE data as the direct restructuring of the global mass matrix would be too complex.

\section{Center of mass velocities of the structure parts to be set to rigid}

When transforming a flexible part into a rigid body, the nodal velocities of the initially flexible part have to be represented by the velocities and angular velocities of the center of mass of the new rigid body. This also involves the reduction of the velocity field. The velocity of an arbitrary point $i$ of the rigid body results from the translational velocity $\dot{\boldsymbol{r}}^{s}$ and the angular velocity $\omega^{s}$ of the rigid body with the vector pointing from the center of mass to the considered point $\boldsymbol{v}_{i}$, as follows

$\dot{\boldsymbol{u}}_{i}=\dot{\boldsymbol{r}}^{s}+\boldsymbol{\omega}^{s} \times \boldsymbol{v}_{i}$

If the mass center velocities are directly computed from the nodal velocities using Eq. (25), strongly varying results depending on the selection of the nodes are obtained. The occurrence of high-frequency vibrations with very small amplitudes may lead to considerable deviations of the nodal velocities from the velocities of a rigid body. An analysis using Eq. (25) that is based on several arbitrarily selected nodes will therefore under normal conditions not fulfill linear and angular momentum balance between the original flexible state and the modified rigid state.

\subsection{Conservation of linear and angular momentum}

The above observations suggest to include linear and angular momentum conservation while determining the mass center velocities for the system in a consistent fashion. This leads to a $6 \times 6$ equation system for each rigid body. Then, linear and angular momentum after the setting to rigid can be computed either by means of the mass center velocities and the rigid body data or through the mass matrix of the system before the setting to rigid and the mass center velocities converted on the nodes of the original discretization, see Eq. (25).

Thus we get for the total structure:

Linear momentum conservation ${ }^{v} \boldsymbol{L}={ }^{n} \boldsymbol{L}$

Angular momentum conservation ${ }^{v} \boldsymbol{J}={ }^{n} \boldsymbol{J}$

with the index $v$ top left referring to values immediately before the setting to rigid and $n$ accordingly to values after the setting to rigid. As the global mass matrix of a flexible system discretized with FE with $n$ nodes can also be represented by $n^{2} 3 \times 3$ node mass matrices $\boldsymbol{M}_{i j}$ with $i, j \in 1, \ldots, n$, for a flexible system with $n$ nodes, linear and angular momentum are given as:

$$
\begin{aligned}
\boldsymbol{L} & =\sum_{i=1}^{n} \sum_{j=1}^{n} \boldsymbol{M}_{i j} \dot{\boldsymbol{u}}_{j} \\
\boldsymbol{J} & =\sum_{i=1}^{n}\left(\boldsymbol{x}_{i} \times \sum_{j=1}^{n} \boldsymbol{M}_{i j} \dot{\boldsymbol{u}}_{j}\right) .
\end{aligned}
$$

When setting to rigid only the nodal velocity vectors of the nodes of the subsequent rigid bodies change. For this reason, only the pertaining portions have to be compared concerning Eqs. (28) and (29). Thus Eqs. (26) and (27) may be reduced to 


$$
\begin{aligned}
& \sum_{i=1}^{n} \sum_{j=1}^{n r b} \sum_{r=1}^{n_{r}^{j}} \boldsymbol{M}_{i r}{ }^{v} \dot{\boldsymbol{u}}_{r}=\sum_{i=1}^{n} \sum_{j=1}^{n r b} \sum_{r=1}^{n_{r}^{j}} \boldsymbol{M}_{i r}{ }^{n} \dot{\boldsymbol{u}}_{r} \\
& \sum_{i=1}^{n}\left(\boldsymbol{x}_{i} \times \sum_{j=1}^{n r b} \sum_{r=1}^{n_{r}^{j}} \boldsymbol{M}_{i r}{ }^{v} \dot{\boldsymbol{u}}_{r}\right) \\
& =\sum_{i=1}^{n}\left(\boldsymbol{x}_{i} \times \sum_{j=1}^{n r b} \sum_{r=1}^{n_{r}^{j}} \boldsymbol{M}_{i r}{ }^{n} \dot{\boldsymbol{u}}_{r}\right) .
\end{aligned}
$$

The index $r$ is a pointer from node $r$ to the currently considered rigid body $j$ with $n_{r}^{j}$ nodes. $n r b$ rigid bodies shall exist in the system. The sum can be split up over all subsequent rigid body nodes in the above double sum as no direct coupling between the rigid bodies is allowed in the proposed strategy as outlined in Section 1. Thus every nodal velocity $\dot{\boldsymbol{u}}_{r}$ of rigid body nodes can be assigned uniquely to one rigid body.

The nodal velocities ${ }^{v} \dot{\boldsymbol{u}}_{r}$ are known. The unknown nodal velocities ${ }^{n} \dot{\boldsymbol{u}}_{r}$ will be expressed with Eq. (25) through the unknown velocities $\dot{\boldsymbol{u}}^{s}$ resp. angular velocities $\omega^{s}$ of the rigid bodies. Now Eqs. (30) and (31) are transformed into

$$
\begin{aligned}
\sum_{j=1}^{n r b} & {\left[\sum_{i=1}^{n} \sum_{r=1}^{n_{r}^{j}} \boldsymbol{M}_{i r}\left(\dot{\boldsymbol{u}}_{j}^{s}+\boldsymbol{\omega}_{j}^{s} \times \boldsymbol{v}_{r}^{j}\right)\right] } \\
= & \sum_{j=1}^{n r b}\left[\sum_{i=1}^{n} \sum_{r=1}^{n_{r}^{j}} \boldsymbol{M}_{i r}{ }^{v} \dot{\boldsymbol{u}}_{r}\right], \\
\sum_{j=1}^{n r b} & {\left[\sum_{i=1}^{n}\left(\boldsymbol{x}_{i} \times \sum_{r=1}^{n_{r}^{j}} \boldsymbol{M}_{i r}\left(\dot{\boldsymbol{u}}_{j}^{s}+\boldsymbol{\omega}_{j}^{s} \times \boldsymbol{v}_{r}^{j}\right)\right)\right] } \\
= & \sum_{j=1}^{n r b}\left[\sum_{i=1}^{n}\left(\boldsymbol{x}_{i} \times \sum_{r=1}^{n_{r}^{j}} \boldsymbol{M}_{i r}{ }^{v} \dot{\boldsymbol{u}}_{r}\right)\right]
\end{aligned}
$$

$v_{r}^{j}$ refers to the vector from the mass center of the subsequently created rigid body $j$ to the considered node $r$.

The equation system consisting of (32) and (33) can now be evaluated separately for each rigid body. In each case, an equation system with 6 equations and 6 unknowns results.

\subsubsection{Flexible system with consistent mass matrix}

As Eq. (33) can be determined separately for each rigid body, a different reference point for the determination of the global angular momentum can consequently be selected for each rigid body. If the center of mass of the current rigid body is selected in the respective equation, then with the afore-mentioned definition for the running index $r$, the equation system for the rigid body $j$ reads:

$$
\begin{gathered}
\sum_{i=1}^{n} \sum_{r=1}^{n_{r}^{j}}\left(\begin{array}{cc}
\boldsymbol{M}_{i r} & \boldsymbol{M}_{i r}\left(\hat{\boldsymbol{v}}_{r}^{j}\right)^{\mathrm{T}} \\
\hat{\boldsymbol{v}}_{i}^{j} \boldsymbol{M}_{i r} & \hat{\boldsymbol{v}}_{i}^{j} \boldsymbol{M}_{i r}\left(\hat{\boldsymbol{v}}_{r}^{j}\right)^{\mathrm{T}}
\end{array}\right)\left(\begin{array}{c}
\dot{\boldsymbol{u}}_{j}^{s} \\
\boldsymbol{\omega}_{j}^{s}
\end{array}\right) \\
=\sum_{i=1}^{n} \sum_{r=1}^{n_{r}^{j}}\left(\begin{array}{c}
\boldsymbol{M}_{i r}{ }^{v} \dot{\boldsymbol{u}}_{r} \\
\hat{\boldsymbol{v}}_{i}^{j} \boldsymbol{M}_{i r}{ }^{v} \dot{\boldsymbol{u}}_{r}
\end{array}\right) .
\end{gathered}
$$

The $6 \times 6$ equation system to be solved is in general filled and unsymmetrical. $\hat{v}_{i}^{j}$ is the skew symmetric tensor defined in Eq. (6).

Remark. With Eq. (34), the rigid body data for a part with $n$ nodes can be developed from the consistent mass matrix of this part. Then the index $i$ runs exclusively over rigid body nodes. The matrix on the left side describes for such a part the mass matrix for an arbitrary reference point $b . v_{j}$ refers to the vector from this reference point to node $j$. The general equation for the mass matrix becomes:

$$
\begin{aligned}
\boldsymbol{M}_{\mathrm{tot}} & =\sum_{i=1}^{n} \sum_{j=1}^{n}\left(\begin{array}{cc}
\boldsymbol{M}_{i j} & \boldsymbol{M}_{i j}\left(\hat{\boldsymbol{v}}_{j}^{b}\right)^{\mathrm{T}} \\
\hat{\boldsymbol{v}}_{i}^{b} \boldsymbol{M}_{i j} & \hat{\boldsymbol{v}}_{i}^{b} \boldsymbol{M}_{i j}\left(\hat{\boldsymbol{v}}_{j}^{b}\right)^{\mathrm{T}}
\end{array}\right) \\
& =\left(\begin{array}{cc}
m^{s} \mathbf{1}_{3 \times 3} & \mathbf{0}_{3 \times 3} \\
\mathbf{0}_{3 \times 3} & \boldsymbol{I}^{s}
\end{array}\right) .
\end{aligned}
$$

Now, the rigid body data can be found through a direct comparison of the components. As the nodal mass matrices of the consistent mass matrix have the form $\boldsymbol{M}_{i j}=m_{i j} \mathbf{1}_{3 \times 3}$, the comparison of the matrix elements on top left in Eq. (35) results directly in:

$m_{s}=\frac{1}{3} \sum_{i=1}^{n} \sum_{j=1}^{n} m_{i j}$.

A comparison of the portions on top right resp. on bottom left in Eq. (35) leads to the following equation after replacing $\boldsymbol{v}_{i}^{b}=\boldsymbol{x}_{i}-\boldsymbol{x}^{s}$ for the center of mass as reference point:

$\boldsymbol{x}^{s}=\frac{\sum_{i=1}^{n} \sum_{j=1}^{n} m_{i j} \boldsymbol{x}_{j}}{m_{s}}$.

From the portion on bottom right in Eq. (35) directly follows:

$\boldsymbol{I}^{s}=\sum_{i=1}^{n} \sum_{j=1}^{n} \hat{\boldsymbol{v}}_{i}^{s} \boldsymbol{M}_{i j}\left(\hat{\boldsymbol{v}}_{j}^{s}\right)^{\mathrm{T}}$.

From this equation the advantages of the selection of the center of mass as reference point become obvious providing as well the reduction of the entries of the secondary diagonals in the mass matrix to the smallest possible values as also the decoupling of the portions for displacements and rotations. If the rigid body is, however, combined and coupled with flexible elements with consistent mass matrices, coupling terms between displacements and rotations become unavoidable. 


\subsubsection{Flexible system with diagonal mass matrices}

When using diagonal mass matrices, the mass matrices of the rigid bodies are decoupled from those of the remaining structure. In this case, the rigid body data can be directly accounted for in the left side of (34). With the mass $m_{j}^{s}$ and the inertia tensor $\boldsymbol{I}_{j}^{s}$ of the rigid body $j$ as well as the nodal mass $m_{r}$ for the rigid body node $n_{r}^{j}$ before the setting to rigid, the following equation results:

$$
\left(\begin{array}{cc}
m_{j}^{s} \mathbf{1}_{3 \times 3} & \mathbf{0}_{3 \times 3} \\
\mathbf{0}_{3 \times 3} & \boldsymbol{I}_{j}^{s}
\end{array}\right)\left(\begin{array}{c}
\dot{\boldsymbol{u}}_{j}^{s} \\
\boldsymbol{\omega}_{j}^{s}
\end{array}\right)=\sum_{r=1}^{n_{r}^{j}}\left(\begin{array}{c}
m_{r}^{v} \dot{\boldsymbol{u}}_{r} \\
m_{r} \hat{\boldsymbol{v}}_{i}^{j v} \dot{\boldsymbol{u}}_{r}
\end{array}\right) .
$$

Then the equation system is decomposed into two decoupled systems, a $3 \times 3$ equation system and three equations with one unknown each.

\section{Energy conservation within the total process}

If a part of a flexible structure is set to rigid, e.g. at time $t_{1}$, the state of deformation is frozen at this point in time, and the strain energy of this part of the structure is stored in the newly generated rigid body. The kinetic energy is transformed to the new rigid body as discussed in Section 5. If this part is reset to flexible later in the analysis, e.g. at time $t_{2}$, the strain energy stored in the rigid body can be completely reconstructed and be restored to the part of the structure that has become flexible again. The kinetic energy is then retransferred with the help of Eq. (25). Within the following subsections we discuss under which pre-conditions this method provides for the conservation of the total energy throughout the total process of setting to rigid and resetting to flexible.

Now the situation is described that a flexible part will be transformed into a rigid body $j$ at time $t=t_{1}$. The velocity of a node $r$ of this flexible part is then ${ }^{v_{1}} \dot{\boldsymbol{u}}_{r}$. This material point has now, immediately after the setting to rigid using linear and angular momentum conservation, the velocity ${ }^{{ }_{1}} \dot{\boldsymbol{u}}_{r}$. In general, it differs slightly from the velocity ${ }^{v_{1}} \dot{\boldsymbol{u}}_{r}$ of the initially flexible node, as the strain rates in the part to be set to rigid are not exactly zero. They are in compliance with the criterion (24) very small but, however, unequal to zero.

The difference velocity

$$
{ }^{t_{1}} \Delta \dot{\boldsymbol{u}}_{r}={ }^{{ }_{1}} \dot{\boldsymbol{u}}_{r}-{ }^{n_{1}} \dot{\boldsymbol{u}}_{r}={ }^{{ }_{1}} \dot{\boldsymbol{u}}_{r}-\left(\dot{\boldsymbol{u}}_{j}^{s}+\boldsymbol{\omega}_{j}^{S} \times \boldsymbol{v}_{j}^{r}\right)_{t=t_{1}}
$$

can now be stored for the time $t_{1}<t<t_{2}$. It describes the relative velocity of the nodes between the flexible and rigid state at the time state of the modification. The rigid body $j$ is then e.g. retransformed into a flexible part at time $t=t_{2}$. Then immediately after the setting back to flexible the velocity vector for the node $r$ becomes:

$$
\begin{aligned}
{ }^{n_{2}} \dot{\boldsymbol{u}}_{r} & ={ }^{v_{2}} \dot{\boldsymbol{u}}_{r}+{ }^{t_{2}} \Delta \dot{\boldsymbol{u}}_{r} \\
& =\left(\dot{\boldsymbol{u}}_{j}^{s}+\boldsymbol{\omega}_{j}^{s} \times \boldsymbol{v}_{j}^{r}\right)_{t=t_{2}}+\boldsymbol{R}_{t_{1} \rightarrow t_{2}}{ }^{t_{1}} \Delta \dot{\boldsymbol{u}}_{r} .
\end{aligned}
$$

In this equation, the relative velocities are rotated with the rigid body; $\boldsymbol{R}_{t_{1} \rightarrow t_{2}}$ refers to the rotation tensor of the rigid body between the times $t_{1}$ and $t_{2}$. In the following section we will find out under which conditions this method can ensure the conservation of the total energy of the flexible structure before and after the setting to rigid. For this the following pre-conditions are assumed:

(1) Energy conservation shall be ensured for the analysis of the structure with the rigid body in the time interval $t_{1}<t<t_{2}$ due to the time integration method selected for this procedure.

(2) If a flexible part of the structure with $\Delta \dot{\boldsymbol{u}}_{r}=\mathbf{0}$ for all nodes, i.e. with a purely static behavior, is set to rigid and reset to flexible, energy conservation is fulfilled for the conditions before and after the setting to rigid. (In the rigid state the strain energy is stored in the rigid body!)

Because of pre-condition (2) ensuring the conservation of the strain energy, proof must be furnished that the difference of kinetic energies when setting to rigid at time $t_{1}$ is as large as the corresponding energy difference when setting to flexible at time $t_{2}$. If it can be shown, that adding the difference velocity $\Delta \dot{\boldsymbol{u}}_{r}$ to the (recomputed) nodal velocities of the rigid body leads to a difference in energy that only depends on $\Delta \dot{\boldsymbol{u}}_{r}$ (and is therefore independent of the nodal velocities of the rigid body that can change between $t_{1}$ and $t_{2}$ ), the desired proof is established. In addition we have to note that the phase correlation between the rigid body velocities and the nodal velocities is lost.

\subsection{Flexible system with diagonal mass matrix}

In the case of diagonal mass matrices for the flexible body parts, the diagonal mass matrices of the subsequently created rigid bodies are neither coupled to each other nor to the mass matrices of the flexible parts. For this reason, the modification of the kinetic energy that occurs when flexible parts are set to rigid can be computed separately for each rigid body. The modification of the kinetic energy $\Delta K$ during the transformation of a part with $n$ nodes into a rigid body at time $t$ becomes then

$$
\begin{aligned}
\Delta K & =\frac{1}{2} \sum_{r=1}^{n}\left[m_{r}\left({ }^{t} \dot{\boldsymbol{u}}_{r}+{ }^{t} \Delta \dot{\boldsymbol{u}}_{r}\right) \cdot\left({ }^{t} \dot{\boldsymbol{u}}_{r}+{ }^{t} \Delta \dot{\boldsymbol{u}}_{r}\right)-m_{r}{ }^{t} \dot{\boldsymbol{u}}_{r} \cdot{ }^{t} \dot{\boldsymbol{u}}_{r}\right] \\
& =\frac{1}{2} \sum_{r=1}^{n}\left[m_{r}\left({ }^{t} \Delta \dot{\boldsymbol{u}}_{r} \cdot{ }^{t} \Delta \dot{\boldsymbol{u}}_{r}+2{ }^{t} \dot{\boldsymbol{u}}_{r} \cdot{ }^{t} \Delta \dot{\boldsymbol{u}}_{r}\right)\right] \\
& =\frac{1}{2} \sum_{r=1}^{n} m_{r}{ }^{t} \Delta \dot{\boldsymbol{u}}_{r} \cdot{ }^{t} \Delta \dot{\boldsymbol{u}}_{r}
\end{aligned}
$$

with the nodal velocity vectors $\dot{\boldsymbol{u}}_{r}$ resulting from the rigid body velocity and the velocity increment ${ }^{t} \Delta \dot{\boldsymbol{u}}_{r}$ 
representing the difference from $\dot{\boldsymbol{u}}_{r}$ to the velocity of the former flexible part. The mixed term in the second line of Eq. (42) disappears, as we can write with the vector $v_{r}$ from the center of mass to node $r$ and the respective velocity vectors ${ }^{t} \dot{\boldsymbol{u}}^{s}$ and ${ }^{t} \omega^{s}$ of the center of mass:

$$
\begin{aligned}
\sum_{r=1}^{n} m_{r}{ }^{t} \dot{\boldsymbol{u}}_{r} \cdot{ }^{t} \Delta \dot{\boldsymbol{u}}_{r}= & \sum_{r=1}^{n} m_{r}\left({ }^{t} \dot{\boldsymbol{u}}^{s}+{ }^{t} \boldsymbol{\omega}^{s} \times \boldsymbol{v}_{r}\right) \cdot{ }^{t} \Delta \dot{\boldsymbol{u}}_{r} \\
= & { }^{t} \dot{\boldsymbol{u}}^{s} \cdot \sum_{r=1}^{n} m_{r}{ }^{t} \Delta \dot{\boldsymbol{u}}_{r} \\
& +{ }^{t} \boldsymbol{\omega}^{s} \cdot \sum_{r=1}^{n} m_{r}\left(\boldsymbol{v}_{r} \times{ }^{t} \Delta \dot{\boldsymbol{u}}_{r}\right)=\mathbf{0} .
\end{aligned}
$$

This is due to the fact that both sums describe the linear momentum resp. the angular momentum of the rigid part based on the difference velocities of the nodes. As the center of mass velocities have been determined satisfying the momentum conservation when setting to rigid-see Section 5, especially (39), both portions must consequently disappear by definition. As shown above adding up $\Delta \dot{\boldsymbol{u}}_{i}$ to the nodal velocities $\dot{\boldsymbol{u}}_{i}$ of the pertaining rigid body always leads to the same kinetic energy modification regardless of the components of $\dot{\boldsymbol{u}}_{i}$ as far as these describe the velocity condition of a rigid body.

As this is valid without restrictions analogously when adding up the difference velocities after the resolution of the rigid bodies, the described method ensures the conservation of the energy in the total process of setting to rigid and resetting to flexible. It must be noted that the kinetic energy is not modified by the rotation of the vector of the differential velocities. Thus neither linear and angular momentum conservation are affected by the differential velocities, because of the procedure described in Section 5.1.

It should be pointed out, however, that the lumped mass concept leads to an error in the computation of the moment of inertia (see [11]). The absolute values of energy and angular momentum are affected by this error. Nevertheless, conservation of these quantities within a time step is guaranteed, using the 'Energy Momentum Method'.

\subsection{Flexible system with consistent mass matrix}

When using consistent mass matrices for the flexible parts, the subsequent rigid and flexible parts are coupling in the mass matrix. As a consequence, there are coupled terms between the rigid body velocities and the nodal velocities of adjacent still flexible parts, when computing the kinetic energy. As the velocity vectors of these flexible parts cannot be described via the center of mass velocities of the rigid body (25), the terms considered in Eq. (43) will not disappear in this case. The energy frozen when a body is set to rigid is consequently not identical in this case to the energy supplied when resetting to flexible due to the afore-mentioned coupling terms. Therefore, energy conservation in the total process can in general not be achieved when consistent mass matrices are used with the proposed rigidizing procedure.

\section{Numerical examples}

\subsection{Free-flying rotating body}

In the following example, we will examine the dynamic behavior of a system consisting of a block and a cantilever as shown in Fig. 4. Both structural parts consist of the same material, for which a St.VenantKirchhoff material law is used within the analysis.

As no boundary conditions are applied, the characteristics of the proposed criterion concerning constant strains caused by centrifugal forces as well as strain rates caused by elastic behavior can be demonstrated in this example. The specified, constant forces are applied to the structure for the time interval $t=2 s$. The loading of the left block is achieved through identical nodal loads on all nodes on the top resp. bottom. To the cantilever, the force is applied in identical portions to the 20 nodes on the top side next to the free end. Thus the external forces only generate an angular momentum around the $y$-axis. The time integration is realized with the Energy Momentum Method as described in Section 2. For demonstration purposes the rigid bodies are retransformed to flexible parts after $t=500 \mathrm{~s}$.
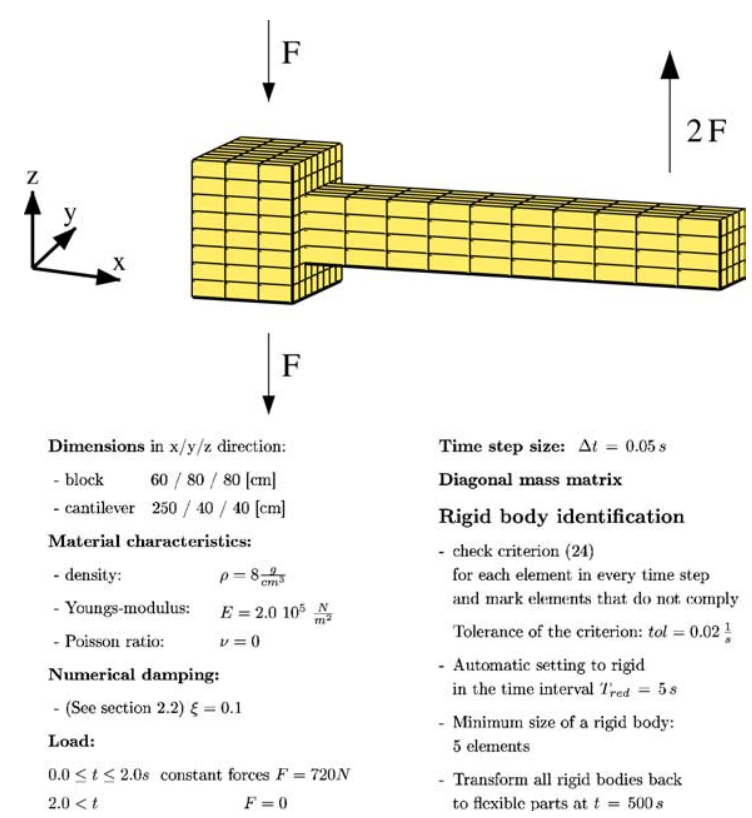

Fig. 4. Free flying rigid body, system, loading, algorithmic information. 

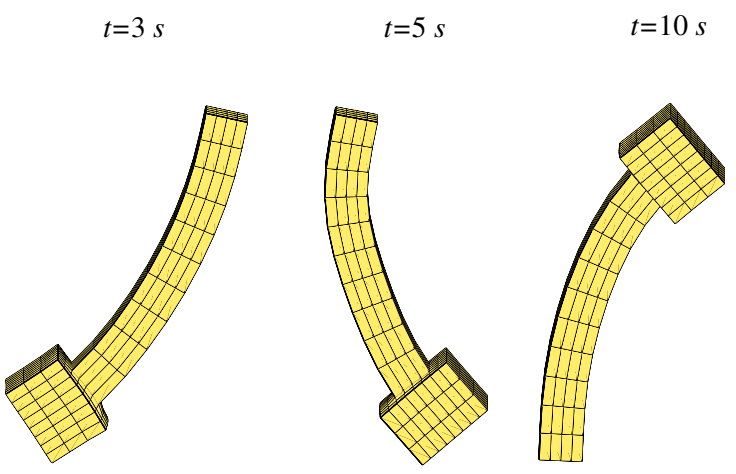

Fig. 5. Deformed structure at defined points in time.

The discussion of the solution focuses on the consideration of the conservation rates as well as the possibility to distinguish between time-independent and time-dependent objective strains with the selected criterion.

As the structure is rather soft, large strains with fairly large strain rates occur immediately after the end of the period during which the external forces were acting, thus at $(t>2.0 \mathrm{~s})$. In Fig. 5 these effects are shown for specific points in time.

The deformation rates decay rapidly as a consequence of the very strong numerical damping. The appli- cation of the rigid body identification check on possible rigid parts in the time interval $T_{\text {red }}$ leads to the successive formation of rigid parts within the structure. Fig. 6 shows the distribution of rigid and flexible parts at particular points in time.

The numerical damping according to Armero and Petöcz [1] applied in this case leads as expected to stronger damping of high-frequency strain rates than of low-frequency ones. The process of setting to rigid is therefore determined by the slow decay of the amplitudes generated by the bending vibrations of the cantilever. These vibrations generate only small strain rates at the free end of the block. Thus, the criterion leads to the first possible rigid bodies in this part. In the transition part between the block and the cantilever, high strain rates occur as a result of the bending vibrations. Therefore, this part remains flexible for almost the complete time interval. The amplitudes of the strain rates resulting from the bending vibrations are smaller at the end of the cantilever than in the transition part to the block. They are also smaller in the center of the cross-sections than on the top or bottom surfaces. This explains the chronological order in which the rigid parts are generated. All elements have complied in all time steps with the criterion given in Eq. (24) between $t=410 \mathrm{~s}$ and $t=415 \mathrm{~s}$ as can be seen in Fig. 6. The flexible elements still present beyond $t=410 \mathrm{~s}$ cannot not be distorted any longer, as all nodes of these elements are already linked to one
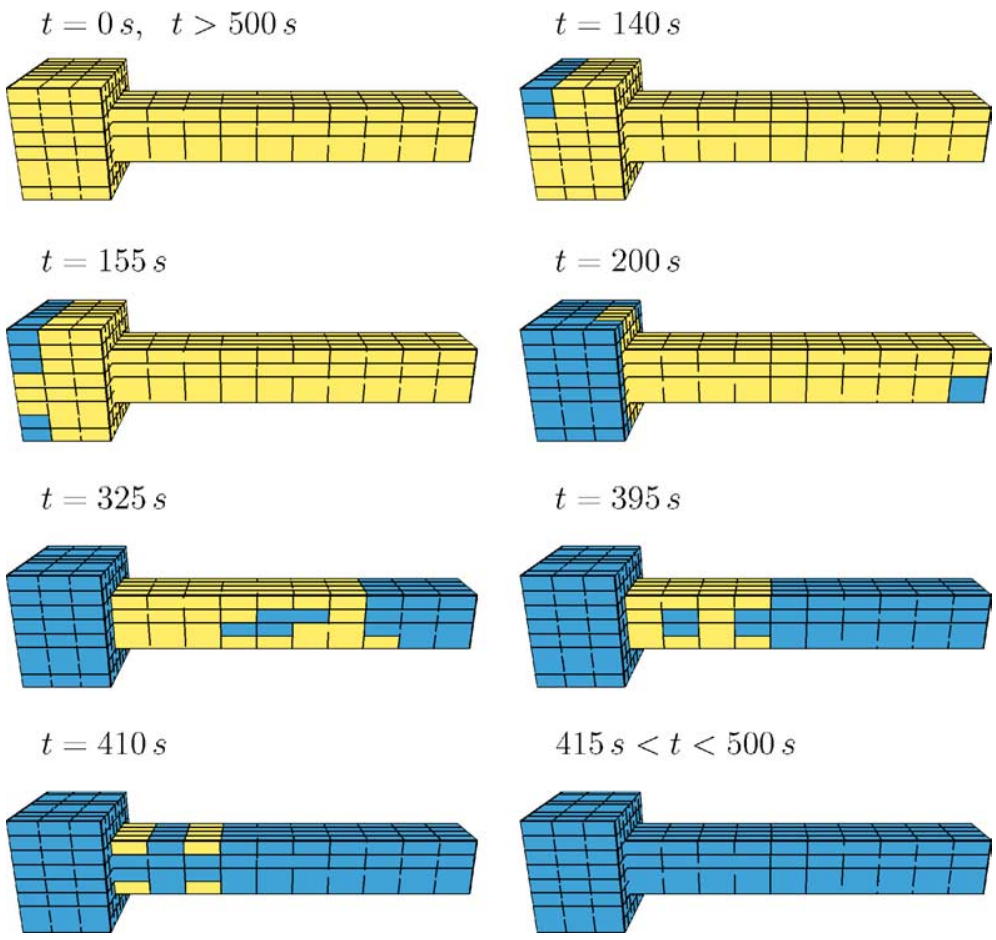

$$
415 s<t<500 s
$$

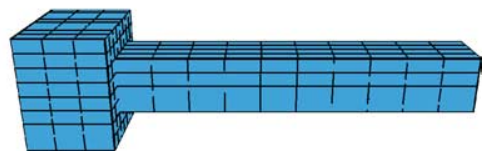

Fig. 6. Evolution of rigid (dark) and flexible (light) parts in the structure. 
single rigid body, i.e. the degrees of freedom of the system for $t>410 \mathrm{~s}$ are already reduced to the 6 degrees of freedom of the rigid body.

As the structure still has stationary strains, even after the decay of the strain rates, resulting from the mass forces generated by the rotation, these strains are frozen in the rigid body for the case of a transformation back to flexible. This takes place at $t=500 \mathrm{~s}$. The selection of the parameters for the rigid-body criterion should be performed such that the relatively slow strain changes resulting from the cantilever vibration can be detected. Therefore, the observation time $T_{\text {red }}$ should be selected taking the duration of the period of this vibration into account.

The energy curves in Fig. 7 show the very large change between kinetic energy and strain energy after the end of the rather short period during which the forces acted upon the structure. The total energy decreases rapidly during this phase as a result of the numerical damping. However, the formation of the first rigid parts is not visible in this figure. These parts are located furthest away from the axis of rotation and therefore, contain hardly any time-dependent strains resulting from the rotation of the total structure. The parts close to the axis of rotation containing the major portion of the time independent strains, are set to rigid in the time domain $300 \mathrm{~s}<t<415 \mathrm{~s}$. Therefore, a relatively high quantity of strain energy is taken out from the system in the latter phase.

Kinetic and total energy are therefore finally identical for the rotating rigid body $(415 \mathrm{~s}<t<500 \mathrm{~s})$. The kinetic resp. strain energy stored in the rigid body as well

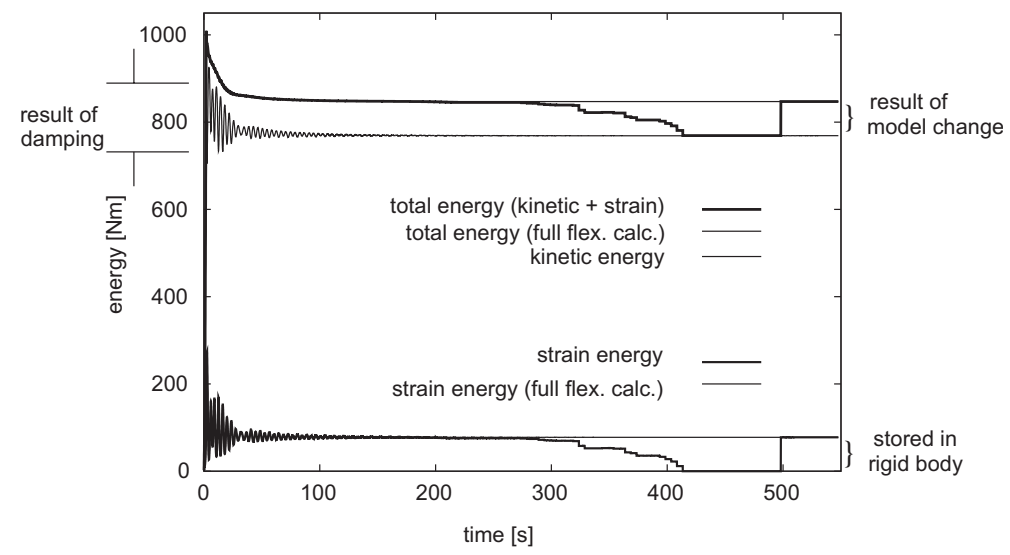

Fig. 7. Energy-time diagram for the total structure, comparing energies for fully flexible and partially rigidized structure.

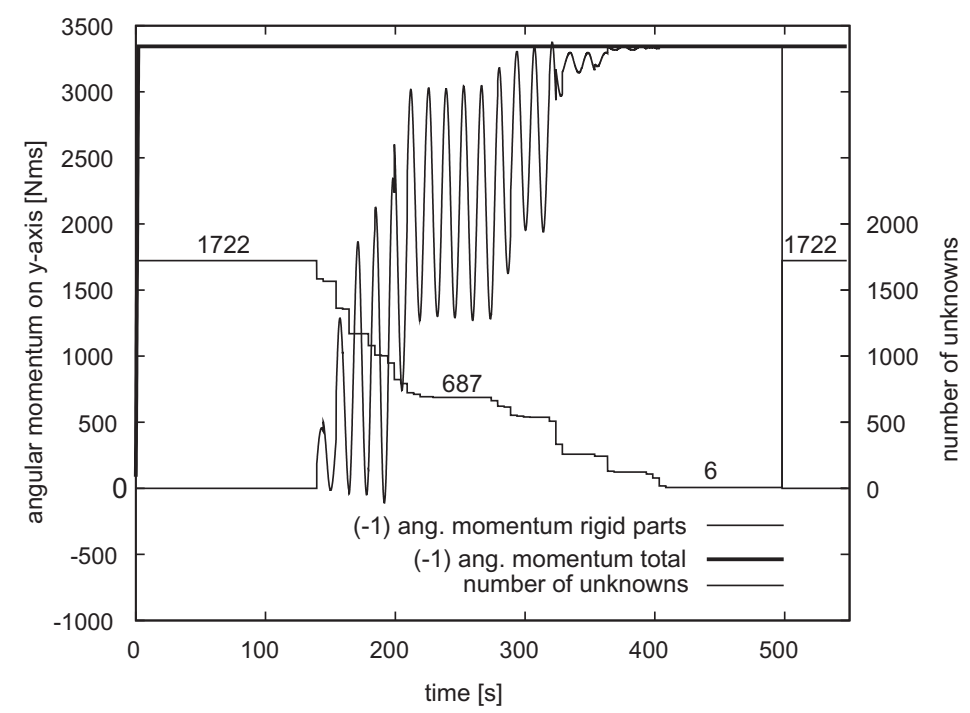

Fig. 8. Angular momentum versus time for the total structure and number of unknowns. 
of the results between an analysis with and without rigid parts shows that neither the energies nor the angular momenta are significantly affected by the proposed method. So the physical behavior is computed correctly although the computational expense is decreasing significantly.

\subsection{Spatial frame with horizontal shock load}

In this example, we will examine the dynamic behavior of a frame structure consisting of a plate and four columns. Again St.Venant-Kirchhoff material law is used within the analysis.

The frame is loaded by a constant uniform surface load acting on the side of the plate in $x$-direction for $0.0 \mathrm{~s} \leqslant t \leqslant 0.05 \mathrm{~s}$ as shown in Fig. 9. As structure and loading pattern are symmetric, symmetry boundary conditions are applied and only half of the system is analyzed. To ensure numerical stability, the Energy Momentum Method described in Section 2 is chosen in combination with numerical damping according to Armero and Petöcz [1]. Due to the high natural frequencies of this very stiff structure the time step size needs to be rather small $(\Delta t=0.01 \mathrm{~s})$ in order to compute the strain rates with sufficient accuracy.

The following discussion focuses on the increase of efficiency within the analysis, the error, occurring from switching to rigid and the possibilities to study and visualize the kinematic behavior of the oscillating structure.

In Fig. 10 the influence of the checking criterion (24) on the total energy and the number of unknowns is depicted. The oscillation of the energy curves is caused by the horizontal vibration in $x$-direction which is the first excited natural frequency. This vibration leads to changing strain rates which again cause corresponding changes in the damping behavior. As the vibration energy is frozen in the rigid body, the fully elastic analysis (crit $=0.0$ ) shows the highest energy level. Obviously higher values of the tolerance criterion lead to an earlier creation of rigid elements and to a larger error in total energy as the part of the energy that is 'frozen' within the rigid bodies increases. The lower end of the curves is determined by the maximum number of rigid elements within the structure.

Focussing on the curves for the tolerance criterion crit $=0.00005$ which show a very small energy error, the first rigid part appears after $t=1.2 \mathrm{~s}$. From this time step to the end of the analysis at $t=2.4 \mathrm{~s}$, the computation using rigid bodies only needs $18 \%$ of the time for a fully elastic analysis.

At $t=2.4 \mathrm{~s}$ only the elements located at a Dirichlet boundary condition remain elastic to prevent the development of kinematic chains. The latter are suppressed within the proposed procedure as the solution of kinematic chains with a master slave concept is very inefficient. This is also shown in Fig. 11 at the symmetry boundary conditions in the plate. Here the development of rigid parts due to the decay of energy is presented. As the numerical damping leads to stronger damping of high frequency strain rates the first rigid parts appear at the center of the plate. The dominating horizontal vibration leads to a hinge like situation at the top and the bottom of the columns. Therefore the middle parts of the columns begin to turn to rigid. The process is finished when the number of rigid bodies is reduced to one and all elements except the ones connected to the boundaries are rigid.

In order to compare the dynamic behavior with and without rigid parts, amplitude spectra for one reference

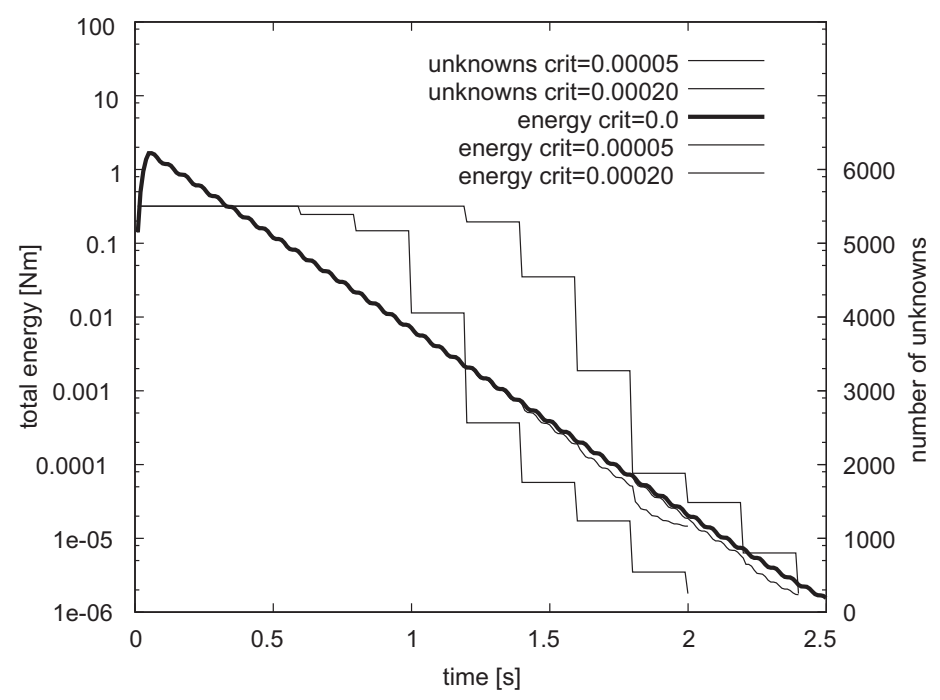

Fig. 10. Energy versus time and number of unknowns for the total structure, varying the tolerance for the rigidity criterion. 
Front view
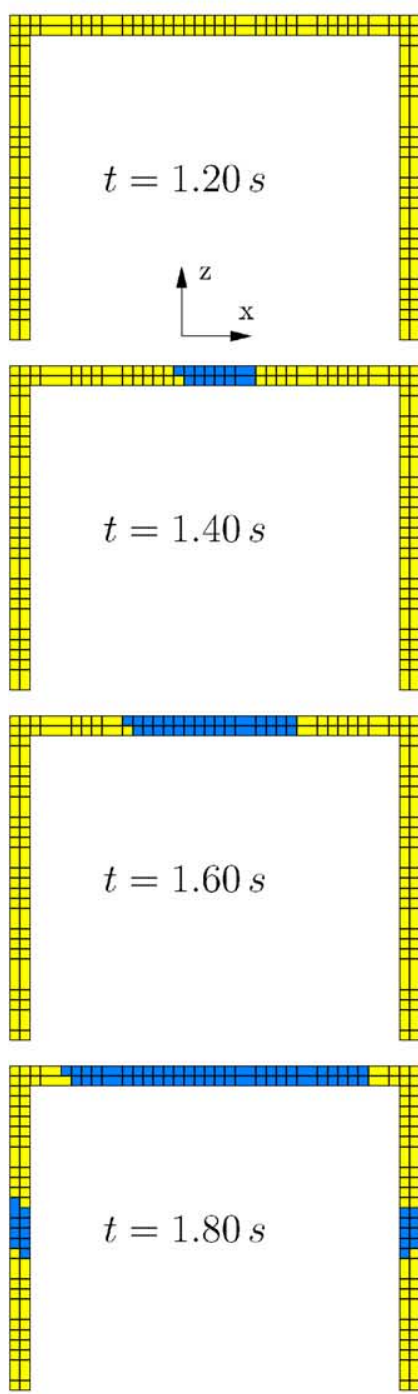

$t=2.20 \mathrm{~s}$
Top view
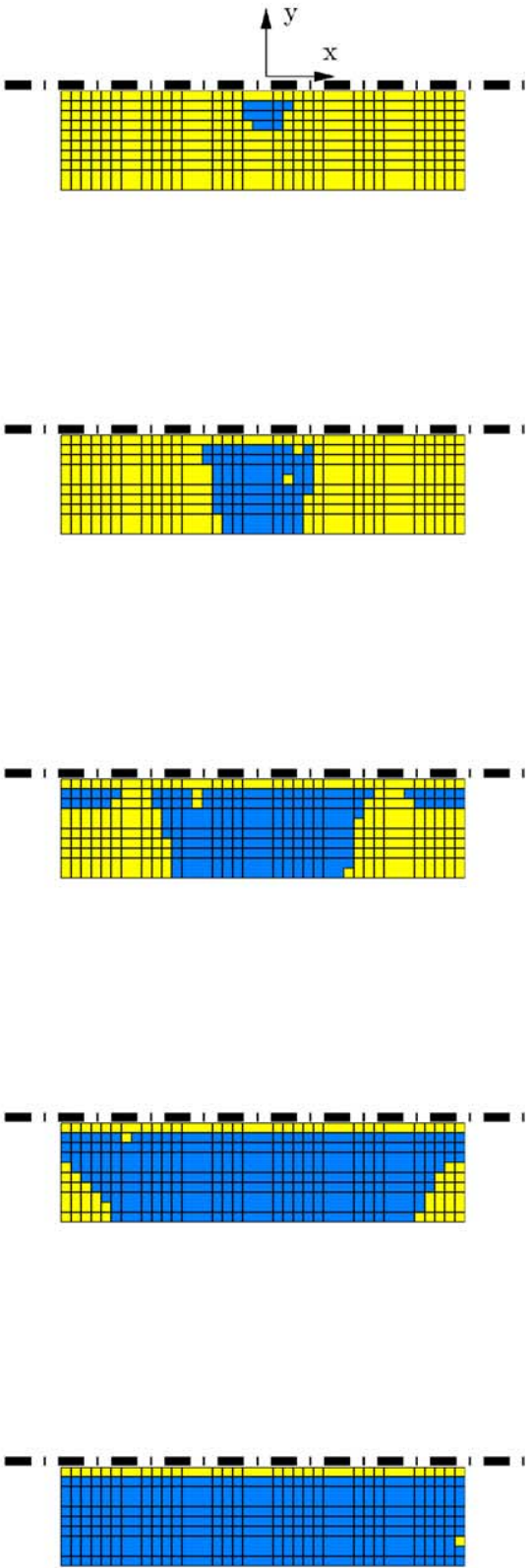

point ' $a$ ' at the frame are presented in Fig. 12. At time $t=2.20 \mathrm{~s}$ the damping in an analysis with switching to rigid and in a fully elastic one is set to zero. The displacement of the reference point in $x$-direction is computed for another eight seconds using a time step size
$\Delta t=0.002 \mathrm{~s}$. A Fourier transformation on the displacements computed with a transient analysis within $2.20 \mathrm{~s} \leqslant t \leqslant 10.20 \mathrm{~s}$ leads to the presented spectrum.

As expected, after $t=2.20 \mathrm{~s}$ only the horizontal vibration is left. Using rigid bodies, the frequency of 


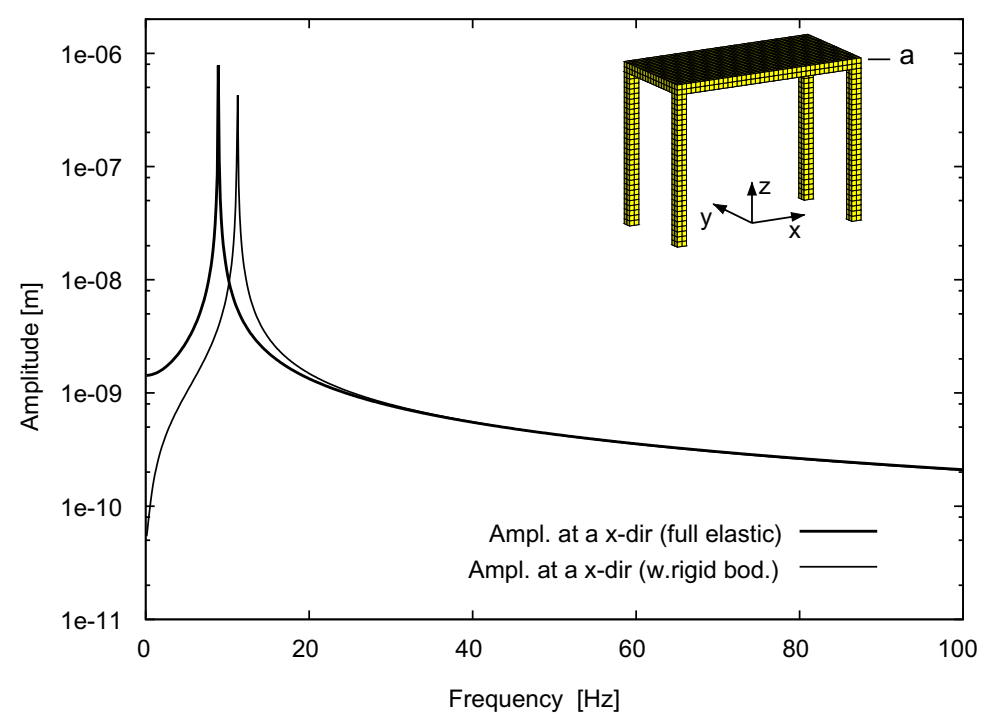

Fig. 12. Amplitude spectrum for $x$-motion at reference point 'a' at $t=2.20 \mathrm{~s}$.

the first mode has increased as parts with small strain rates have lost their flexibility being modified to rigid. The decay of the amplitude is caused by the loss of strain energy within the rigid parts, combined with the higher stiffness. Obviously the dominant dynamic behavior concerning the $x$-motion in point a is not significantly altered by the switching to rigid.

\section{Conclusions}

The intention of this contribution is to develop a procedure that allows the automatic identification of parts in flexible structures that can be treated in the subsequent analysis approximately like rigid bodies as a direct consequence of their mechanical behavior. In order to find the appropriate model, the flexible parts of the structure are analyzed during a defined period of time on compliance with e.g. the proposed criterion that is based on strain rates. If the strain rates of a particular part of the structure are below a certain threshold during the considered period, the part is set to rigid for the subsequent analysis. The compliance with mechanical conservation laws when changing the model is of particular importance in this context. The proposed method allows the fulfillment of linear and angular momentum conservation for the total system. As the strains of the flexible parts that should be set to rigid are in general not equal to zero the energy conservation is fulfilled at the setting to rigid, only if the strain energy is somehow stored in the rigid body. We have to note that though the criterion is based on small strain rates, the strains themselves do not necessarily have to be small. If the rigid body is subsequently transformed back into a flexible part, energy conservation can be achieved in the total process of modifying the model twice. To achieve this, the respective strains as well as the relative velocities in the rigid body have to be stored as additional rigid body data. The numerical - fairly simpleexamples show that the developed procedure performs very well.

\section{References}

[1] Armero F, Petöcz E. Formulation and analyses of conserving algorithms for frictionless dynamic contact/ impact problems. Comput Meth Appl Mech Eng 1998;158:269-300.

[2] Chen AJ. Energy-momentum conserving methods for three dimensional dynamic nonlinear multibody systems. $\mathrm{PhD}$ thesis, Division of mechanics and computation, Stanford University, 1998.

[3] Garcia Orden JC, Goicolea JM. Non-frictional contacts in multibody dynamics with conserving schemes. In: ECCM 1999, European Conference on Computational Mechanics, Munich, Germany, 1999.

[4] Garcia Orden JC, Goicolea JM. Conserving properties in constrained dynamics of flexible multibody systems. Multibody Syst Dyn 2000;4:225-44.

[5] Garcia Orden JC, Goicolea JM, Arribas JJ. Conserving properties in multibody dynamics with the penalty method. In: Computational Mechanics, New Trends and Applications, Barcelona, Spain, 1998.

[6] Göttlicher B. Effiziente Finite-Element-Modellierung gekoppelter starrer und flexibler Strukturbereiche bei transienten Einwirkungen. PhD thesis, Institut für Mechanik, University Karlsruhe, 2002.

[7] Hauptmann R, Schweizerhof K. A systematic development of solid-shell element for linear and nonlinear analysis employing only degrees of freedom. Int J Numer Meth Eng 1998;42:49-70. 
[8] Ibrahimbegovic A, Mamouri S. On rigid components and joint constraints in nonlinear dynamics of flexible multibody systems employing $3 \mathrm{~d}$ geometrically exact beam model. Comput Meth Appl Mech Eng 2000;188:805-32.

[9] Ibrahimbegovic A, Mamouri S. Energy conserving/decaying implicit time-stepping scheme for nonlinear dynamics of three-dimensional beams undergoing finite rotations. Comput Meth Appl Mech Eng 2002;191:4241-58.

[10] Ibrahimbegovic A, Mamouri S, Taylor RL, Chen AJ. Finite element method in dynamics of flexible multibody systems: Modeling of holonomic constraints and energy conserving integration schemes. Multibody Syst Dyn 2000;4:195-224.

[11] Ibrahimbegovic A, Taylor RL, Lim H. Non-linear dynamics of flexible multibody systems. Comput Struct 2003;81: 1113-32.
[12] Miehe C, Schröder J. Energy and momentum conserving elastodynamics of a non-linear brick-type mixed finite shell element. Int J Numer Meth Eng 2001;50: 1801-23.

[13] Simo JC, Tarnow N. The discrete energy-momentum method. Conserving algorithms for nonlinear elastodynamics. ZAMP, Z Angew Math Phys 1992;43:75793.

[14] Simo JC, Wong KK. Unconditionally stable algorithms for rigid body dynamics that exactly preserve energy and momentum. Int J Numer Meth Eng 1991;31:19-52.

[15] Taylor RL, Chen AJ. Numerical solution of coupled rigid and flexible systems. In: Proc IACM World Conference, Buenos Aires, 1998.

[16] Zienkiewicz OC, Taylor RL. The finite element method, vol. 2. Butterworth-Heinemann; 2000. 